\title{
HYBRID VOLTAGE REGULATOR FOR AUTOMOBILES
}

\author{
AKIHIRO SAWAMURA, TADASHI TAKAHASHI and SEI-ICHI DENDA \\ Sanken Electric Co., Ltd., 3-6-3 Kitano, Niiza, Saitama, Japan
}

\begin{abstract}
In the course of the development of hybrid voltage regulators for automobile use, increasing the reverse blocking voltage with decreasing of saturation voltage for output power darlington transistors, the capability of monolithic IC chip against surge pulses generated in cars, and an improvement of the pattern design of thick film in order to withstand temperature cycling, have been important subjects. Power darlingtons have more than $150 \mathrm{~V}$ of reverse voltage as well as large secondary breakdown capacity and less than $1.2 \mathrm{~V}$ of saturation voltage. A monolithic chip having small saturation voltage and more than $500 \mathrm{~mA}$ of surge current capability, has been developed. A neck-shaped thick film pattern was developed to make soldering in a controlled manner. The problems in the earlier stage with surge current and temperature cycling have been almost solved by these improvements.

In this paper the characteristics of the transistor and the monolithic chip, the structure of the device and related problems observed in temperature cycling are described.
\end{abstract}

\section{INTRODUCTION}

A hybrid voltage regulator for automobile use was developed ten years ago in Sanken Electric and at present the regulators are in mass production with the aid of semiconductor technology improvement, such as the success of flip-chip small signal device and well-passivated solderable high power transistor.

In spite of rather long history IC regulators just started to replace conventional mechanical regulators three years ago. The reason of the delay would be due to low reliability expected and expensiveness. Since all the problems have been settled at present, the mechanical regulators would be totally replaced by the electronic counterparts in the very near future because of their small size, light weight, high current capability, free from maintenance and high reliability.

\section{CIRCUIT}

There are several standard circuit configurations for voltage regulators. Though a variety of voltage sensing and feedback connection have been reported fundamental circuits are all alike. For our device a simpler circuit is being used as shown in Figure 1. The main reason of adopting the simple one is to improve reliability and reduce manufacturing cost. The circuit consists of just four elements except for resistors, DTr, Ds, MIC, and Cn. The components in the dotted line are integrated in a semiconductor chip.

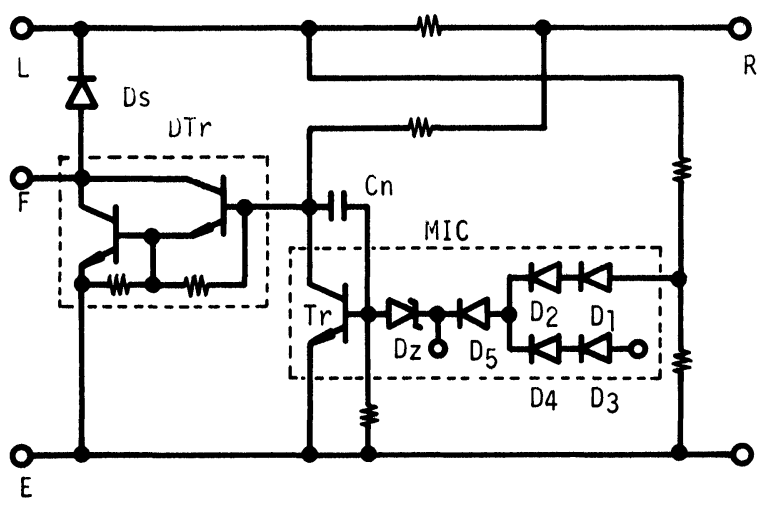

FIGURE 1 Circuit of hybrid IC regulator.

Conventionally the temperature dependency of regulator was compensated by the combination of a discrete thermistor, silicon diodes, and a zener diode but this is controlled by the elements in the chip in IC regulator.

\section{POWER DARLINGTON AND MONOLITHIC CHIP}

The high voltage surge which is generated in cars has caused problems for electronic devices so far. IC regulator is no exception. The improvements of the darlington and the monolithic is essentially required. 


\subsection{Darlington Transistor}

There are two types of surge pulse, an ignition surge and a load-dumped surge. In the former pulse time is rather short though high voltage is often observed. On the contrary low voltage and longer time, namely total electrical energy is large in the latter. $\mathrm{BV}_{\mathrm{CEO}}$ of the power darlington must be higher than the surge which occurs in a full load dump condition, so that the darlington transistor in Figure 1 can be operated safely without adopting a protective circuit element like an avalanche diode.

The surge voltage being generated when the full load is dumped, is measured about $150 \mathrm{~V},{ }^{1}$ in the case of the alternator rotation at $10,000 \mathrm{rpm}$. The ignition surge voltage of above $300 \mathrm{~V}$ is often observed. The saturation voltage of the darlington transistor must be small enough from the stand-point of alternator efficiency as well as decreasing power loss. In conclusion a transistor having the characteristics of high blocking voltage and small saturation voltage at the same time, and small chip size to obtain lower production cost are required. The transistors in production have more than $150 \mathrm{~V}$ of blocking voltage and less than $1.2 \mathrm{~V}$ (at $I c=4 \mathrm{~A}$ ) of saturation voltage. Chip size is $5 \mathrm{~mm} \times 5 \mathrm{~mm}$.

It is usually difficult to obtain a transistor which satisfies both the saturation voltage and cost, and having a higher blocking voltage than the ignition surge pulse. Although the ignition surge is high, its energy is relatively small. Accordingly the transistor was designed as not going into secondary breakdown area though the blocking voltage is lower than the surge.

The darlington transistor is shown in Figure 2 and its cross section in Figure 3 respectively. The emitter-base and the base-collector junctions are glass-passivated, so the transistor surface is stable enough against humidity. Since all electrodes are $\mathrm{Ni}$ plated, lead wires can be

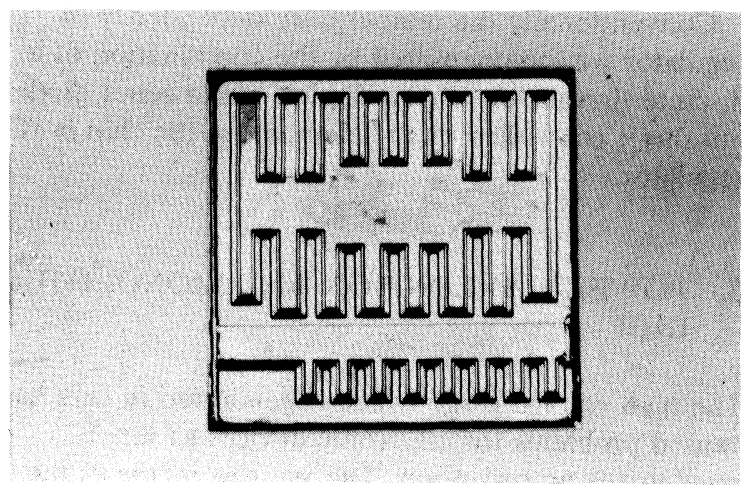

FIGURE 2 Power darlington transistor.

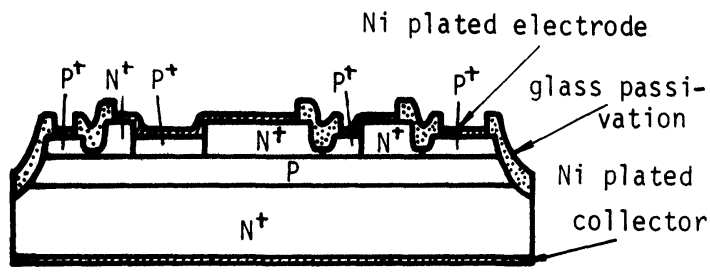

FIGURE 3 Cross section of glass passivated power darlington transistor.

soldered to the electrodes directly. The Ni electrode has advantages in cost and pull strength over other multi-layer electrode and aluminium electrode.

\subsection{Monolithic Chip}

In regards to the monolithic chip, it is important that the saturation voltage of transistor Tr must be small. Although the transistor usually operates at about $10 \mathrm{~mA}$, the saturation voltage at this current would not be a problem, but when the load dumped surge is applied to the regulator, the saturation voltage goes high at about $200 \mathrm{~mA}$. If this saturation voltage is larger than the forward drop of base-emitter of the darlington, the transistor can be operated and may be destroyed by excessive power loss.

Secondly the current capability from diode $D_{1}$ to the emitter of transistor $\mathrm{Tr}$ is important when the surge is applied. When the surge current in Figure 4 is applied

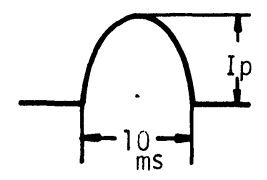

FIGURE 4 Surge current waveform.

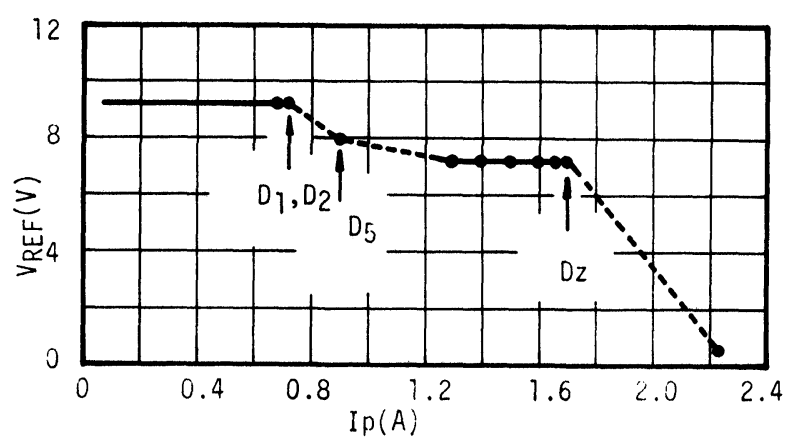

FIGURE 5 Surge capacity measurement, $V_{\text {REF }}$ decreases with Ip. 
to the diode $D_{1}$ and to the emitter of transistor $\operatorname{Tr}$, the variation of the potential at diode $D_{1}$ namely $V_{\mathrm{REF}}$ is shown in Figure 5.

Since the current capacity of the diode $D_{1}$ is smallest, it will be burned out first. But if its capacity is above $500 \mathrm{~mA}$, it is no problem for the ignition or load dump surge. The monolithic chip has flip-chip electrode with six bumps. The chip is shown in Figure 6. The zener diode pattern is designed round to prevent current concentration and the surface of the chip is covered with sputtered $\mathrm{SiO}_{2}$ to be stable to humidity and other environmental contaminations.

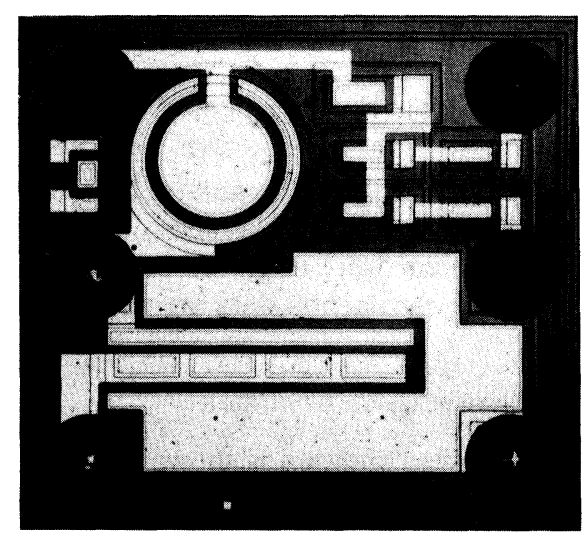

FIGURE 6 Monolithic chip with six solder bumps.

\subsection{Device Structure}

The components in the circuit of Figure 1 are mounted on a ceramic substrate which is shown in Figure 7. Each element is reflow bonded on a solder coated substrate and silver lead wires are connected by soldering. The dimensions of the ceramic substrate is $20 \mathrm{~mm} \times 25$ $\mathrm{mm} \times 0.63 \mathrm{~mm}$ of $96 \%$ alumina. Resistors of $\mathrm{RuO}_{2}$ are

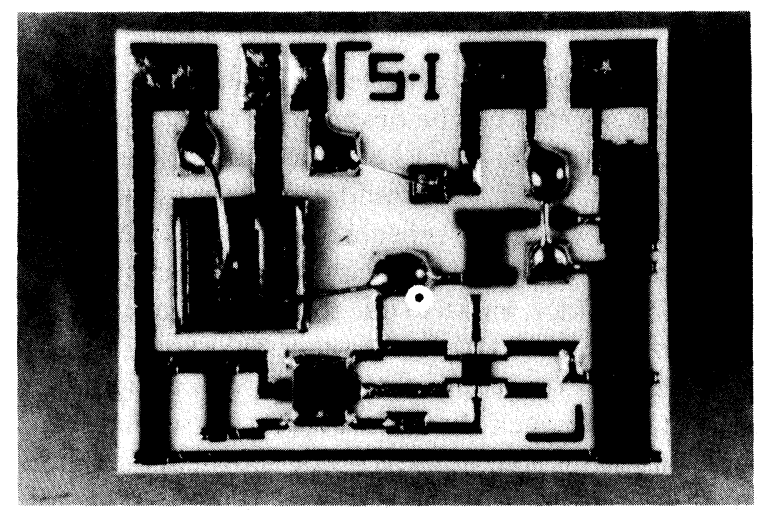

FIGURE 7 Completed substrate assembly. screen printed, first and glass coated. The resistors are stable against high surge voltage and humid condition. Conductors are also glass coated to prevent current leak problems. The cross section of darlington transistor, DTr, diode, Ds, and monolithic chip are shown in Figure $8 \mathrm{a}$ to Figure $8 \mathrm{c}$. The darlington is soldered on Ni-plated $\mathrm{Cu}$ base in order to absorb a transient power loss. Silver lead wires of $0.3 \mathrm{~mm}$ in diameter with nail heads are shown in Figure $8 \mathrm{a}$ and Figure $8 \mathrm{~b}$.

The pull strength of solder bond is much larger than that of wire bonding with gold and aluminum. The pull strength of the figure is above $1 \mathrm{Kg}$ by the reason of thick and nail headed lead wire. The six-bumped monolithic IC is reflow bonded to the substrate pattern on a belt reflow furnace. Solder wetting takes place at the same time for all the component electrodes and bumps giving uniform bonding and accordingly high reliability in severe mechanical environment.
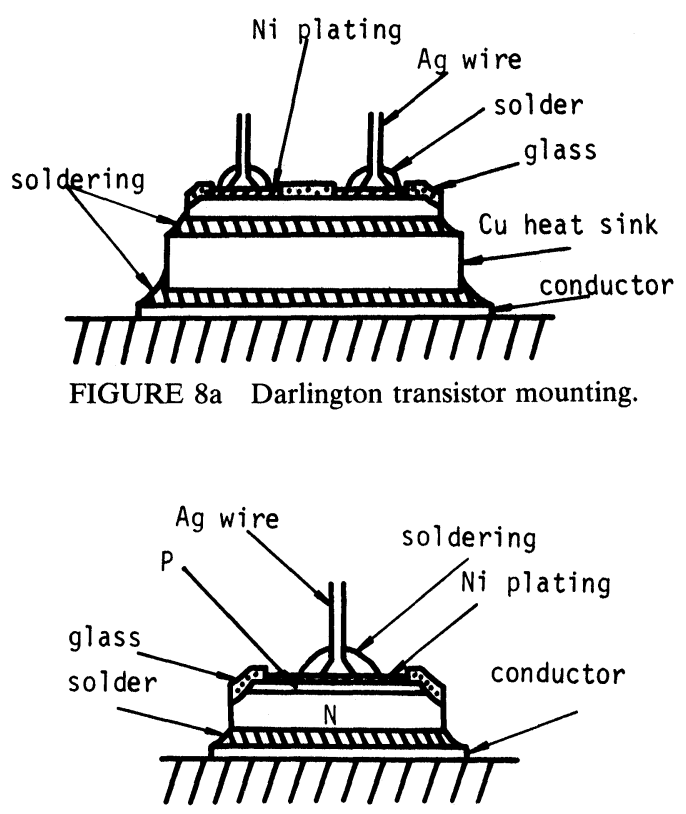

FIGURE 8b Diode mounting.

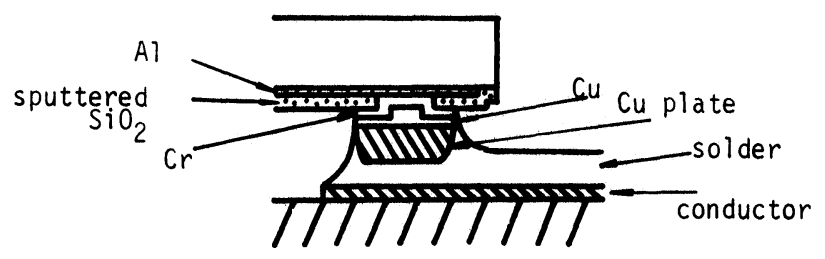

FIGURE 8c Flip chip bonding. 


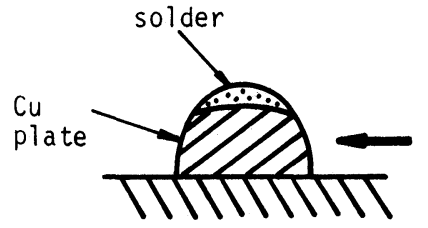

FIGURE 9 Bump shear strength measurement.

The pull strength of bonded wire has been reported about $15 \mathrm{~g}^{2}$ for gold wire. The shear strength of a bump shown in Figure 9 is measured above $150 \mathrm{~g}$, ten times larger than gold wire bonding. Measured distribution is shown in Figure 10.

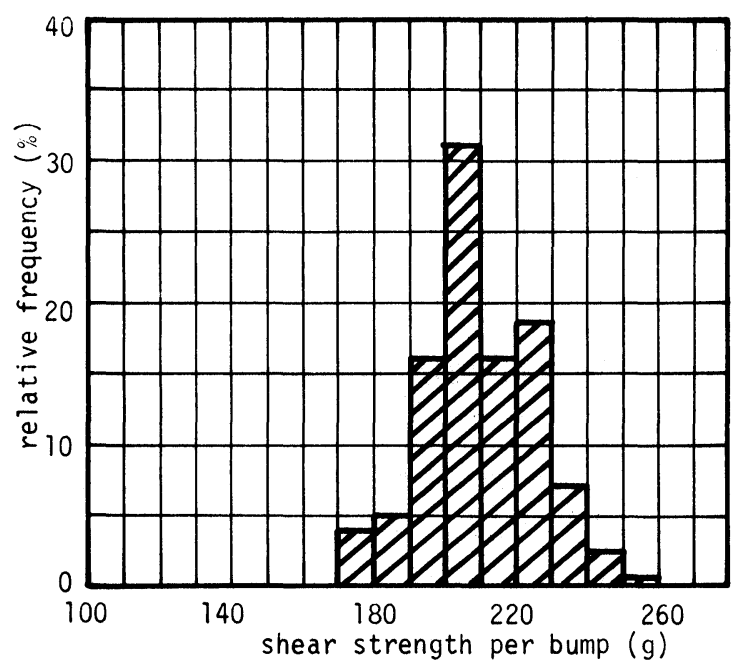

FIGURE 10 Measured bump shear strength.

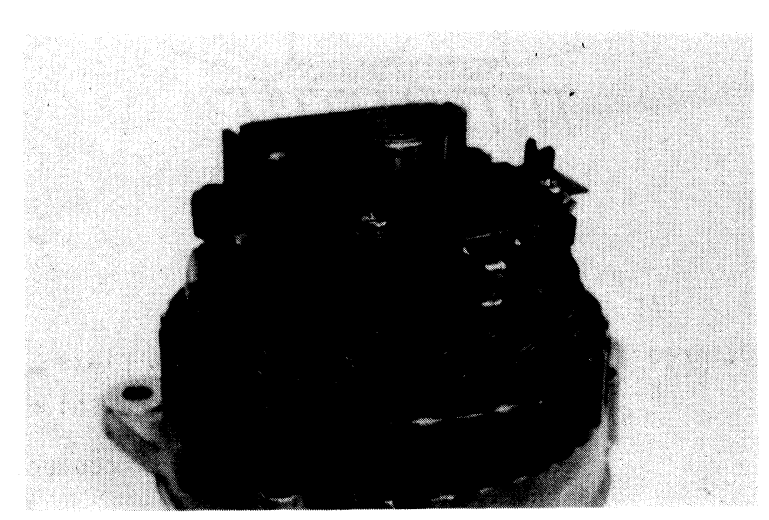

FIGURE 11 Installed regulator in alternator.
Each element and a cross over wire are coated with silicone rubber to absorb mechanical stress caused by thermal expansion difference and to protect semiconductor surface from contaminations. After functional trimming for regulating voltage setting the substrate is attached to an aluminum heat absorber and potted by resin. The regulator is then installed inside the alternator as shown in Figure 11.

These structures can be deduced to be the most suitable to operate in specially severe temperature variation and mechanical vibration in automobile application. This feature has been proved in the field for several years.

\section{TEMPERATURE CYCLING}

One of the problems of an IC regulator became clear by performing life test. The small cracks or break in the thick film conductor were observed by temperature cycle test. The experimental data are as follows.

\subsection{Temperature Cycle Condition}

Temperature cycle condition is shown in Figure 12. It takes less than 10 seconds for the sample container to move between low and high temperature chamber.

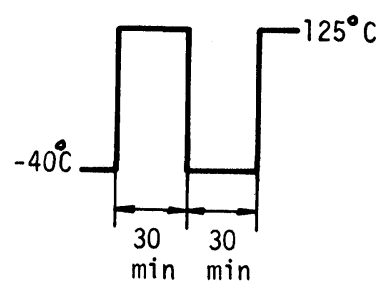

FIGURE 12 Temperature cycling condition.

\subsection{Crack or Breaking of Thick Film Conductor}

When the temperature cycle test is repeated more than 300 times, a crack can be observed at the steep step as point A shown in Figure 13 and the value of the resistor will increase. The crack would grow to a break as the temperature cycling is repeated. This is due to the poor pattern design of conductor. The steep step is formed by solder, and also due to the fact the lead wire $B$ is too straight for absorbing thermal expansion and compression. Therefore it is necessary to design the pattern with a neck shown in Figure 14, so that soldering forms a moderated shape shown in the figure. In addition by making the lead wire $B$ more loopy, the crack does not occur until 600 times of cycling. 


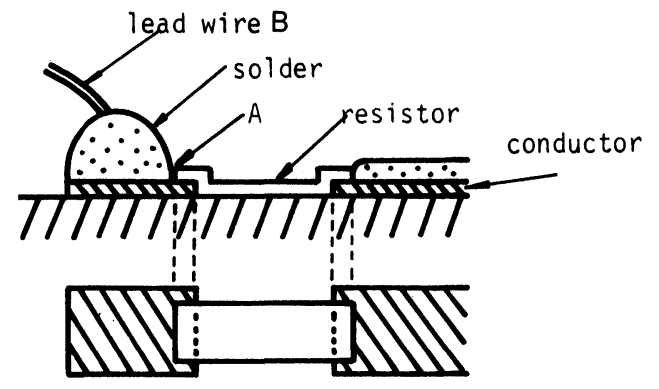

FIGURE 13 Soldering pad design (I).

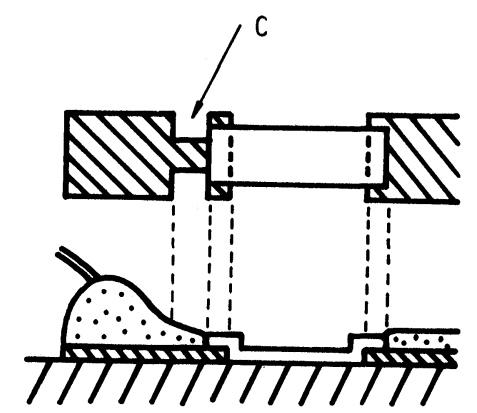

FIGURE 14 Soldering pad design (II).

\section{FUTURE}

The field failure rate of conventional mechanical regulators is said to be about $0.02 \%$. According to statistics in the market, the IC regulators have kept the level of less than $0.01 \%$ of failure rate. It is believed that the result could accelerate the application of hybrid circuit in automobile industry.

In the near future the alternator field current must be increased in order to take more current out of the generator because higher current will be required for the future automobile. The mechanical regulator will be rapidly replaced for this reason.

Increase of the field current will give necessarily more power loss in the regulator. Stable operation at higher temperature will be required for regulators of the next generation. ${ }^{3,4}$

\section{ACKNOWLEDGEMENTS}

The authors would like to acknowledge the great help and cooperation given by Mr. Iwaki and Mr. Morishita of Mitsubishi Electric Co.

\section{REFERENCES}

1. M.Morishita, "IC regulator",Electronic Parts and Materials, (May 1979).

2. A. Sawamura, "Wire Bonding onto Thick Film Conductor", Sanken Technical Report, (Nov. 1970).

3. Howard W. Imhof and Bruce E. Bertsch, "Thick-film Materials for High Temperature Operation" High Temperature Electronics and Instrumentation Seminar, (Dec. 1979).

4. D. W. Palmer, "Hardening Microcircuits against $300^{\circ} \mathrm{C}$, Yesterday and Tomorrow" High Temperature Electronics and Instrumentation Seminar, (Dec. 1979). 

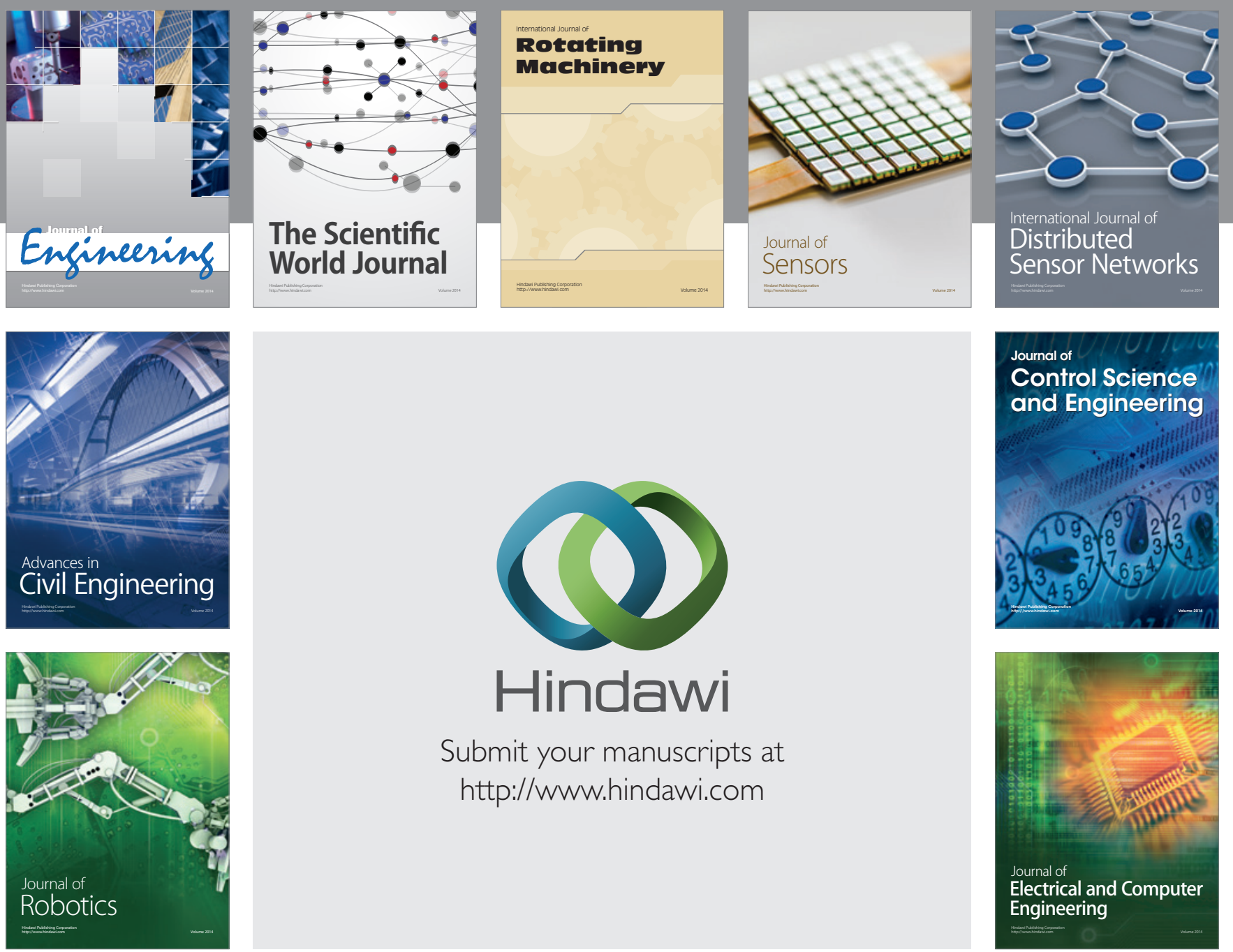

Submit your manuscripts at

http://www.hindawi.com
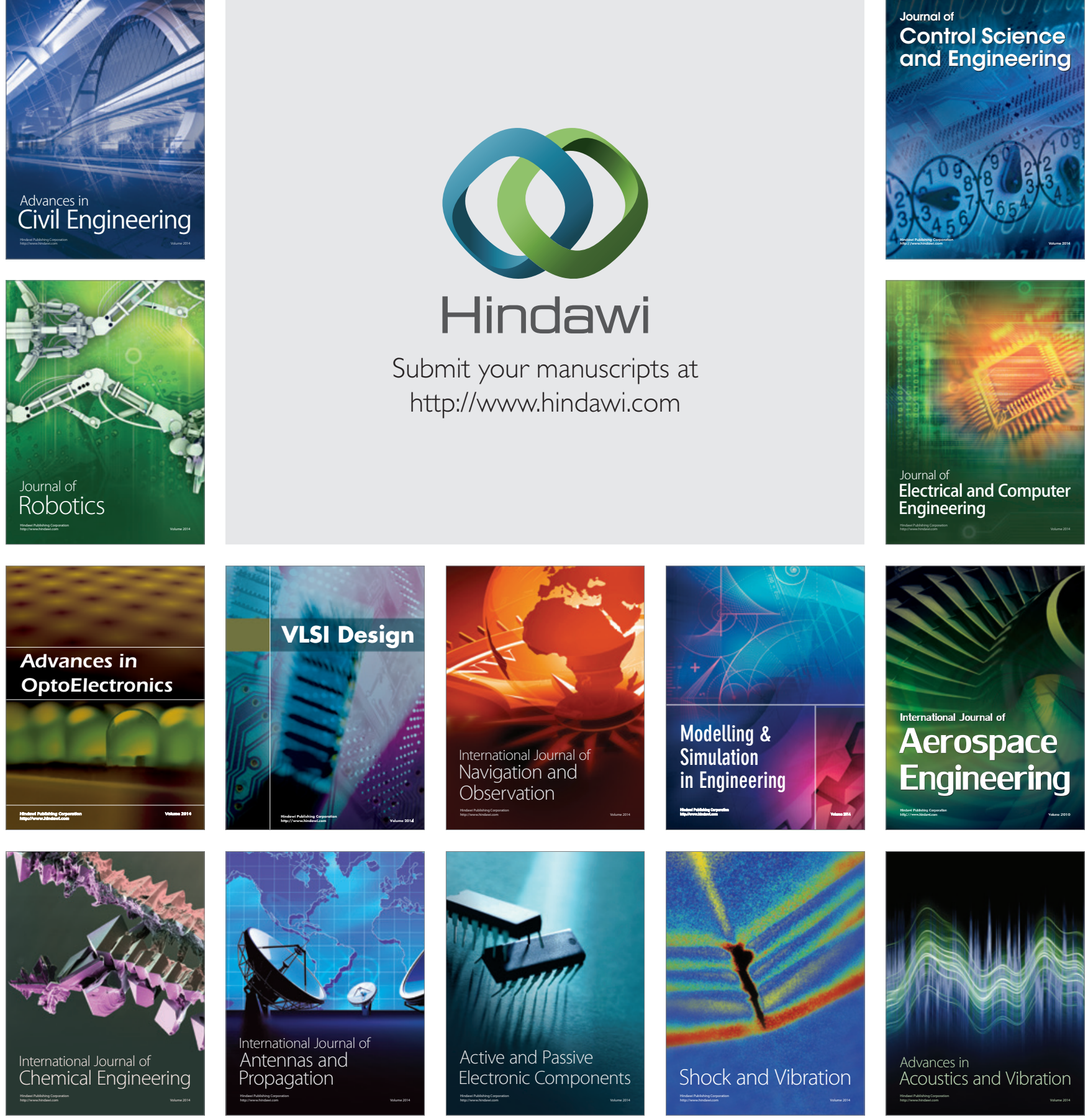